\title{
An Efficient Authentication Scheme for Electronic Health Records based Block Chain
}

\author{
Swati Laxmeshwar \\ Department of Computer Science and Engineering \\ City Engineering College, Bangalore, India
}

\begin{abstract}
This paper presents our current electronic health record ( EHR) systems with a blockchain based architecture.Designed on top of established databases held by health care organizations, the framework incorporates a blockchain approach to ensure data record accuracy and enhance network interoperability by monitoring all incidents that occurt on top of existing health service provider-maintained databases, the architecture implements a blockchain solution to ensure data record integrity and improve system interoperability by tracking all events that occur in database data. We 're also implementing a new reward system in this revised design to build new blocks on the blockchain. The design is autonomous of any particular blockchain network and accessible to more extensions; it may also be combined with other electronic storage structures needing security. It will then theoretically tie in with other electronic storage schemes needing protection from data misuse.
\end{abstract}

\section{INTRODUCTION ${ }^{1}$}

As of late, the blockchain has drawn a great deal of considerations from both scholarly and mechanical fields, and its applications have been infiltrated from budgetary spaces into in a portion of the data and correspondences innovation zones. The idea of blockchain innovation was first presented by Satoshi Nakamoto in the notable paper on the Bitcoin. 1 The blockchain innovation is a veritable blend of existing procedures from disseminated figuring, cryptography, game theory,2,3 and so on, and it vows to fundamentally change the manner in which advanced resources are traded among untrusted members, safely following the responsibility for resources without the control of a focal power.

Distributed computing offers an open door for people and organizations to offload to incredible servers the weight of overseeing a lot of information and performing computationally requesting activities. Because of the expanding notoriety of distributed computing, an ever increasing number of information proprietors are propelled to redistribute their information to cloud servers for incredible comfort and decreased expense in information the board. Information proprietors offer administrations to an enormous number of organizations and organizations, and they stick to high-security norms to improve information security by following a layered methodology that incorporates information encryption, key

\author{
Deepak N.R \\ Department of Computer Science and Engineering \\ City Engineering College, Bangalore, India
}

administration, solid access controls, and security knowledge.

Social insurance is an information serious space where a lot of information is made, spread, put away, and got to day by day. Obviously innovation can assume a critical job in improving the nature of care for patients (e.g., utilizing information investigation to settle on educated clinical choices) and conceivably lessen costs by more effectively assigning assets as far as staff, gear, and so forth.

Electronic Medical Records (EMRs) contain, for the most part, clinical and clinical information identified with the patient and taken away from the competent social insurance provider. This encourages the recovery and investigation of information on social insurance. More easily, the administration of EMRs, early ages of Health Information Systems (HIS) is planned with the ability to make new EMR occurrences, store them, and question and recover away from EMRs of interest. 2 Moderately basic arrangements can be schematically portrayed as a graphical UI or web administration. These are commonly the frontend with a database at the back-end, in a brought together or conveyed execution. With understanding versatility (both inside and remotely to a given nation) being progressively the standard in the present society, it became clear that various independent EMR arrangements must be made interoperable to encourage sharing of medicinal services information among various suppliers, even across national outskirts, varying. For instance, in clinical the travel industry centers, for example, Singapore, the requirement for ongoing social insurance information sharing between various suppliers and across countries turns out to be progressively articulated.

To encourage information sharing or even patient information convenientce, there is a requirement for EMRs to formalize their information structure and the plan of HIS. Electronic Health Records (EHRs), for instance, are intended to permit persistent clinical history to move with the patient or be made accessible to various social insurance suppliers (e.g., from a rustic emergency clinic to a medical clinic in the capital city of the nation, before the patient looks for clinical consideration at another emergency clinic in an alternate country). 3 EHRs have a more extravagant information structure than EMRs. There have additionally been activities to build up HIS and frameworks that can scale and bolster future needs, as confirm by the different national and worldwide activities, for example, the Fascicolo Sanitario Elettronico (FSE) 
venture in Italy, the epSOS venture in Europe, and a continuous task to normalize sharing of EHRs.

The certainty of adroit apps (e.g. Android and iOS devices and wearable devices) has brought about a change of perspective within the therapeutic administration sector, starting late. These tools can be guaranteed or delivered to the consumer by the restorative administration company to assess client performance (e.g. patients) and to prompt / support clinical care and patient view. For example, there is a wide variety of classes of portable (applications) related to well-being, wellness, weight loss, and other human administration. For example, customer enrollment works out / works out, keeps the number of exhausted calories and various experiences ( e.g. number of steps taken, and so on) mainly function as an after instrument.

There are similarly devices with introduced sensors for additional created clinical assignments, for instance, arm groups to measure heartbeat during activities, or contraptions for self-testing of glucose. The data (e.g., customer's basic signs) can be industriously amassed and sent ceaselessly to a splendid device, before being sent to a remote restorative administrations cloud for extra examination. Another model is Ambient Assisted Living responses for therapeutic administrations expected to recognize imaginative telehealth and telemedicine benefits in order to give remote individual health watching.

These improvements have made ready for Personal Health Records (PHR), where patients are increasingly engaged with their information assortment, checking of their health conditions, and so forth., utilizing their cell phones or wearable gadgets (e.g., savvy shirts and keen socks).

Blockchain was initially intended to record exchange information, which is moderately little in size and straight. At the end of the day, one just concerns itself about whether the present exchange can be followed back to the first "deal."

\section{LITERATURE SURVEY}

\section{A. Outsourced symmetric private information retrieval}

Redistributing is the way toward getting a current business process that an association recently performed inside to an autonomous association, where the procedure is bought as a help. The information proprietor empowers SSE Scheme and re-appropriates a record or assortment of documents to a remote server in encoded structure. And furthermore, the information proprietor approves customers (outsiders) to look through the database to learn. Despite everything the remote server does not find out about the information or questioned values as in the basic SSE setting. We expand Cash et al's OXT convention to assist subjective Boolean inquiries in the entirety of the above models while standing up to antagonistic non-conspiring servers (data proprietor and remote server) and selfassertive malignant customers to save the convention 's striking execution.
$>$ Advantages

- We are implementing digital signatures.

- Cryptographic protocols with different security and privacy features.

- We are supporting various signature schemes without adding additional hardware complexity compared to a hardware implementation of a conventional signature scheme.

\section{$>$ Disadvantages}

- Encryption keys are not simple strings of text like passwords.

- Damage is massive when you lost your symmetric key.

\section{B. Dynamic search-able symmetric encryption}

Searchable Symmetric Encoding (SSE) allows a customer to encrypt information so that search tokens can be later created for a capacity server to send requests. We suggest the central SSE program to achieve all the resources such as sub-straight hunting period etc. Expands the transformed list method in a few non-trifling areas, and incorporates new SSE layout approaches. We are implementing our strategy and performing a presentation test, showing that our approach is highly successful and ready for arrangement.

$>$ Advantages

- Security against keyword-adaptive attacks.

- Indexes which are compact.

- Ability to efficiently add and delete files;

\section{$>$ Disadvantages}

- Every means of electronic communication is insecure as it is impossible to guarantee that no one will be able to tap communication channels. So the only secure way of exchanging keys would be exchanging them personally.

\section{Highly-scalable searchable symmetric encryption with support for Boolean queries}

The design, analysis and execution of the primary open symmetric encryption (SSE) convention that supports conjunctive inquiry and general Boolean inquiries on spread equally encoded information and scales to large databases and self-assertive information including free content hunting. Our answer offers a sensible and commonsense exchange between execution and security by effectively supporting huge databases to the re-appropriated serve, at the cost of moderate and all around defined spillage.

\section{Advantages}

- Providing performance results of a prototype applied to several large representative data sets, including encrypted search over the whole English Wikipedia.

- Load Balancing

$>$ Disadvantages

- Exact matching may retrieve too few or too many documents. 


\section{EXISTING SYSTEM AND PRAPOSED SYSTEM}

\section{A. Existing System}

The existing system doesn't securely maintain and process the data. It does not provide the search result which is more accurate. Incorrect and misleading data will produce the result of wrong analysis. Low Efficiency Search. The scheme's search time period is proportional to the database capacity. This isn't appropriate for repositories on broad size.

\section{$>$ Disadvantages}

- Low Achievement Quest.

- The search time of scheme is proportional to the size of the database.

- The large volume repositories are not suitable for this.

- Doesn't support verification of file updates.

- Attacks upon integrity of data.

\section{B. Proposed System}

We are introducing this system to overcome the security problems that occur in the existing system and effectively store the data over the cloud. The owner of data outsources the authenticated information into the cloud.

The data user gets through answer, the proof and the public authentication key, they may verify the freshness, validity, and completeness of the search result without decrypting it.

\section{$>$ Advantages}

- Successful Search results.

- Prevents attacks on freshness and integrity of data;

- Offers high health requirements.

- Easy File Update.

\section{SYSTEM ARCHITECTURE}

Distinctive CA (Certificate Authentication) associations structure a BC (Block Chain) to make and keep up a bound together declaration that is perceived by approval, and to guarantee genuine and powerful information sharing, to guarantee that the genuine information can't be altered. Dispersed hubs are built by BC innovation, which will permit $\mathrm{CA}$ associations in various locales to join the $\mathrm{BC}$ arrange. CAs gives testament issuance and approval through accord component. Accord testaments will be recorded in the $\mathrm{BC}$. These testaments are all $\mathrm{CA}$-endorsed authentications in the $\mathrm{BC}$, and the entire $\mathrm{BC}$ will turn into. A joint declaration confirmation collusion across CA can supplant highlight point CA scaffolds to set up secure correspondence channels between various CA frameworks, which rearranges the board and usage costs.

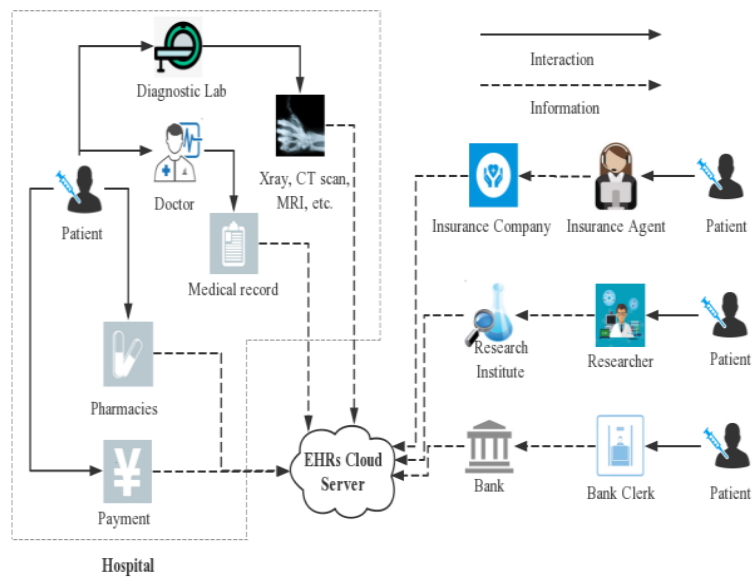

Fig 1:- System Architecture

Anyway when all associations are on a BC, the security of client is difficult to ensure. The genuine name of the declaration holder will be set apart in the BC. At the point when the authentication holder presents the declaration to the specialist co-op or site, the genuine character data of the client will be uncovered. So it is hard to utilize unknown administrations to secure client' $\mathrm{s}$ protection, ,for example, web based exchanging or electronic democratic .We propose a protection mindful PKI framework dependent on consent BCs.

As appeared in Figure 2, the framework comprises of enrollment $\mathrm{BC}(\mathrm{RBC})$, authentication $\mathrm{BC}(\mathrm{CBC})$ and client. The $\mathrm{RBC}$ hub is answerable for client distinguishing proof, scrambling client personality data and putting away verified client information after encryption. The CBC hub is liable for client lawfulness confirmation, and afterward endorsements with verification data and administration data to clients and stores unknown advanced testaments, and stores mysterious computerized authentication information. Furthermore, store mysterious computerized testament information.

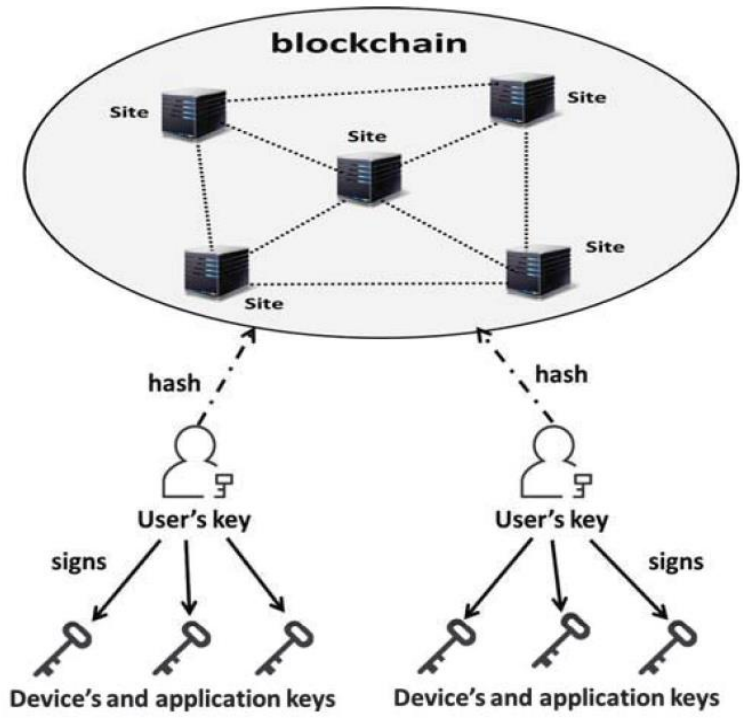

Fig 2:- Key and Block Exchange 


\section{IMPLIMENTATION}

\section{$>$ Initialization}

Suppose healthcare providers $(\mathrm{P})$ agree to share their records by joining the blockchain network. In general, they are assumed to agree to:

- Execution contract and document ties contract;

- Measurement of pertinence and reward mechanism when adding a new block to the chain;

- Blockchain update rates (e.g., regular emergency situations);

- Processes for the creation, verification and introduction of new parts.

Each provider $\mathrm{Pk}, \mathrm{k}=1,2, \ldots, \mathrm{n}$, will be associated at the initialization stage with a $\mathrm{Si}$ significance based on the quantity and value of the health records in their own database. We've specified the quantity and value of the records as: assume $\mathrm{Pk}$ has $\mathrm{qk}$ records in its database, Supplier importance $\mathrm{Pk}$ is identified as

$$
s k=\sum_{t=1}^{q k} v t
$$

Where vt refers to each record value At the Bt. user value For different stakeholders the perception of a record 's importance can vary. Here we propose to describe the importance of a record based on two principles: comprehensiveness and disclaimer. To assess completeness, both providers decide on a list of certain important items in a record in advance and add to the overall value of that record for each important item present in a record. For consistency, we must understand that for records of a specific user, the contents of such things may be replicated by several providers. In that case, the contribution from those items will be less valued than those unique ones..By taking completeness and redundancy into consideration, we define the value of a record as

$v t=\frac{1}{\Omega} \sum_{\omega=1}^{\Omega} A t, \omega, K t, \omega$

Where at, ubiquitous $=1$ if the uppermost object in the database has content and At, ubiquitously $=0$. KING is the redundancy indicator of a given item in a record and is defined as 1 divided by the number of providers with records filled with the same item. The meaning of a provider $\mathrm{Pk}$ is thus given by the

$s k=\sum_{t=1}^{q k}\left(\frac{1}{\Omega} \sum_{\omega=1}^{\Omega} A t, \omega, K t, \omega\right)$

If $A t, \omega=0$ for all $\Omega$ items, it essentially means that the provider $P k$ does not have any value from the $t^{\text {th }}$ record and therefore gets no value of contribution from this record added to its significance. Note that this definition ensures that a record always has value less than or equal to 1 .

\section{CONCLUSION AND FUTURE ENHANCEMENT}

In this paper, we proposed a design that executes blockchain advancement for the reliability of social protection records in national EHR systems and improves the interoperability of the existing structures. Applying without specifying the board's database capacity is appropriate for current e-social security systems and workable for prosperity suppliers. Considering that the mechanism is essentially a separate access system and prosperity providers individually take care of information, we require providers to assume simple blockchain assistance responsibilities, including designing , testing, and adding new block. In this transaction wise arrangements are used and can also be adapted to various preferences, e.g. by adding specific items to the ledger in documents to obey. This heuristic structure is freed from a specific blockchain platform and its assortments can fit various electronic recording systems for access.

\section{FUTURE ENHANCEMENT}

Information Classification dependent on Security: A distributed server farm can store data from various customers. In order to make the safety degree contingent on the quality of information, it should be possible to group information. This ordering plan should take account of various perspectives, such as access recurrence, updating recurrence and access by various elements, etc. If the information is characterized and labeled, security relating to this specific data component can be applied at that point. The security level includes confidentiality, encryption, reliability, capability and so on, which depend on the type of information that is selected.

\section{REFERENCES}

[1]. M.Steward,"Electronic Medical Records," Journal of Legal Medicine, vol. 26, no. 4,2005.

[2]. R. Hauxe, "Health Information Systems Past, Present,Future,"Int'l Journal of Medica Informatics, 2006.

[3]. K. Häyrinena et al., "Definition, Structure, Content, Use and Impacts of Electronic Health Records: A Review of the Research Literature," Int'l Journal of Medical Informatics, vol. 77, no. 5, 2008, pp. 291304.

[4]. M.Ciampi et al.,"A Federated Interoperability Architecture for Health Information Systems," Int' 1 Journal of Internet Protocol Technology, vol. 7, no. 4, 2013, pp. 189-202.

[5]. M. Moharra et al., "Implementation of a Cross-Border Health Service: Physician and Pharmacists' Opinions from the epSOS Project" 2015.

[6]. S.H. Han et al., "Implementation of Medical Information Exchange System Based on EHR Standard" 2010. 
[7]. D. He et al., "A Provably-Secure Cross-Domain Handshake Scheme with Symptoms-Matching for Mobile Healthcare Social Network," IEEE Transactions on Dependableand Secure Computing,

[8]. F.Y. Leu et al., "A Smartphone-Based Wearable Sensors for Monitoring Real-Time Physiological Data," Computers and Electrical Engineering, 2017.

[9]. M. Memon et al., "Ambient Assisted Living Healthcare Frameworks, Platforms, Standards, and Quality Attributes", 2014.

[10]. P.C. Tang et al., "Personal Health Records: Definitions, Benefits, and Strategies for Overcoming Barriers to Adoption" 2006.

[11]. S. Marceglia et al., "A Standards-Based Architecture Proposal for Integrating PatientHealth Apps to Electronic Health Record Systems" Applied Clinical Informatics, 2015.

[12]. A. Mu-Hsing Kuo, "Opportunities and Challenges of Cloud Computing to Improve Health Care Services" Journal of Medical Internet Research, 2011.

[13]. V. Casola et al., "Healthcare-Related Data in the Cloud: Challenges and Opportunities" IEEE Cloud Computing, 2016.

[14]. S. Nepal et al., "Trustworthy Processing of Healthcare Big Data in Hybrid Clouds" IEEE Cloud Computing, 2015.

[15]. G.S. Poh et al., "Searchable Symmetric Encryption: Designs and Challenges" 2017.

[16]. Q. Alam et al., "A Cross Tenant Access Control (CTAC) Model for Cloud Computing: Formal Specification and Verification" IEEE Transactions on Information Forensicsand Security, 2017.

[17]. M. Li et al., "Scalable and Secure Sharing of Personal Health Records in Cloud Computing Using AttributeBased Encryption" IEEE Transactions on Parallel andDistributed Systems, 2016.

[18]. F. Tschorsch and B. Scheuermann, "Bitcoin and Beyond: A Technical Survey on Decentralized Digital Currencies" IEEE Communications Surveys \& Tutorials, 2016.

[19]. A.Azaria et al.,"MedRec:Using Blockchain for Medical Data Access and Permission Management" Proceedings of the 2nd Int' 1 Conference on Open and Big Data, 2016.

[20]. J. Zhang, N. Xue, and X. Huang, "A Secure System for Pervasive Social Network-Based Healthcare", 2016.

[21]. J.McKinlay et al.,"Blockchain:Background, Challenges and Legal Issues," DLA Piper Publications, 2016. 